\title{
Application of Artificial Intelligence for Optimizing an Organic Solar Cells Production Process
}

\author{
Grazia Lo Sciuto \\ Department of Electrical, Electronic and Informatics Engineering, University of Catania, Viale A. Doria 6, 95125 \\ Catania, Sicily, Italy
}

Received April 28, 2020; accepted June 22, 2020; published June 30, 2020

\begin{abstract}
The study of organic solar cells (OSCs) has been rapidly developed in recent years. Organic solar cell technology is sought after mainly due to the ease of manufacture and the cells exclusive properties such as mechanical flexibility, light weight, and transparency. These properties of OSCs are well-suited for unconventional applications with power conversion efficiencies higher than $10 \%$. The flexibility of used substrates and the thinness of the devices make OSCs ideal for roll-toroll production. However, organic solar cells still have very low conversion efficiencies due to degradation and stability of the technology. To extract their full potential, OSCs have to be optimized. On the other hand, the production chain of organic solar cells (OSC) can take advantage of the use of artificial intelligence (AI). In fact, AI integration into the production workflow makes solar cells more competitive and efficient. This paper presents some applications of AI for optimizing the OSCs production processes
\end{abstract}

The efficiency of organic solar cells is primarily limited by the ability of the active layer to absorb all the sunlight. The typical architecture of a polymer solar cell is shown in Fig. 1 and it consists of different layers including an active layer sandwiched between the anode and the cathode with different work functions. Many effects tend to reduce the efficiency of organic solar cells such as variations in the devices length. By investigating the physics of organic devices under atmospheric conditions, the results of simulations and measuring current/voltages, the variation length of the devices is not negligible affecting the recombination of charge carriers in organic solar cells.

The basic design constraints in OSCs significantly influence energy absorption by light trapping and consequent conversion efficiency. OSCs electrical characteristics strongly depend on the device's geometry $[1 \div 2]$. Among such geometrical values, the surface dimension and thickness, particularly the active layer thickness, influence the performance of the solar cell. Therefore, the electrical performance can be related to several parameters such as carrier mobility, donoracceptor ratio, materials concentrations, and the morphology of the blend $[3 \div 4]$.

Artificial intelligence can be easily integrated into the production workflow as a complementary step between the electrical characterization and the production of the organic solar cells. Furthermore, the device accuracy depends on its geometry (height and width) so a modelling approach based on artificial intelligence could be an alternative interest in manufacturing. In this application, a back propagation neural network is developed to disclose the link between the device length and its maximum power output. The results show that the device length influences its efficiency, which is to be considered in the manufacturing processes.

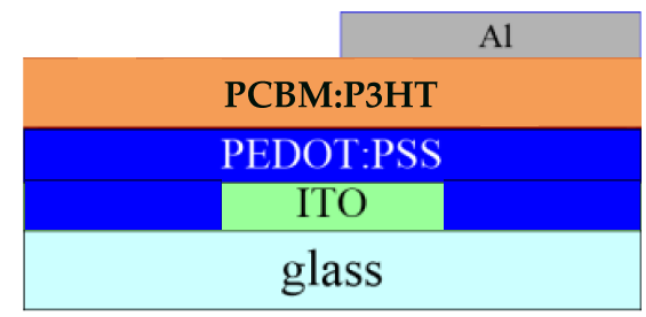

Fig. 1. Structure of the investigated OSC.

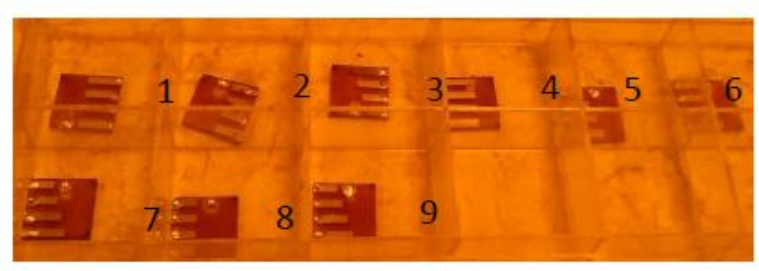

Fig. 2. The realized devices.

The fabrication and testing of Organic Photovoltaic devices have been developed in laboratory, in the Optoelectronic Organic Semiconductor Device Laboratory (OOSDL), Department of Electrical and Computer Engineering at Ben-Gurion University of the Negev. The procedure of fabrication started with the precleaning of indium tin oxide (ITO) coated glass substrates produced by Xin Yan Technology Ltd. by means of sonication (KUDOS HP Series $53 \mathrm{kHz}$ high frequency ultrasonic cleaner) in methanol, acetone, isopropanol alcohol, 15 minutes each and by subsequent rinsing. After this pre-cleaning, $\mathrm{O}_{2}$ plasma treatment is applied to the surfaces to remove the organic particles on the surface. Following the plasma treatment, a thin layer of poly $(3,4-$ ethylenedioxythiophene) polystyrene sulfonate PEDOT:PSS was spin-coated at $5000 \mathrm{rpm}$ for $60 \mathrm{~s}$, after passing through a $4.5 \mu \mathrm{m}$ syringe filter. The 
coated substrate was then baked at $100^{\circ} \mathrm{C}$ for 60 minutes on a hot plate. Next, a mixture of 10mg Poly(3hexylthiophene) $\mathrm{P} 3 \mathrm{HT}$, which is a regioregular semiconducting polymer and $10 \mathrm{mg}$ of fullerene derivative [6,6]-phenyl-C61-butyric acid methyl ester PCBM blend was dissolved in $1 \mathrm{ml}$ Chloroform and stirred for 1 hour in a nitrogen filled glove box. This polymer blend was deposited on top of the sample at $1000 \mathrm{rpm}$ for $60 \mathrm{~s}$ via spin-coating by passing through a $100 \mathrm{ml}$ filter. High temperature annealing treatment was performed in the glove box for $30 \mathrm{~min}$ at $140^{\circ} \mathrm{C}$. In the top contact fabrication, a $80 \mathrm{~nm}$ thick aluminum (Al) layer was subsequently deposited via vacuum thermal evaporation to connect the thin film to an electrical wire. Silver Conductive Epoxy was deposited on a small part of ITO, removed locally, and the aluminum layer. It is electrically conductive offering a strong conductive bond.

Silver Conductive Epoxy, which is a common mixture of bright silver epoxy and grey silver hardener, was mixed and deposited on ITO and the aluminum layer to create bonding adhesion and electrical connectivity.The analyzed polymer solar cells have been made and tested in laboratory realizing 9 devices, each of which contains 4 organic solar cells.

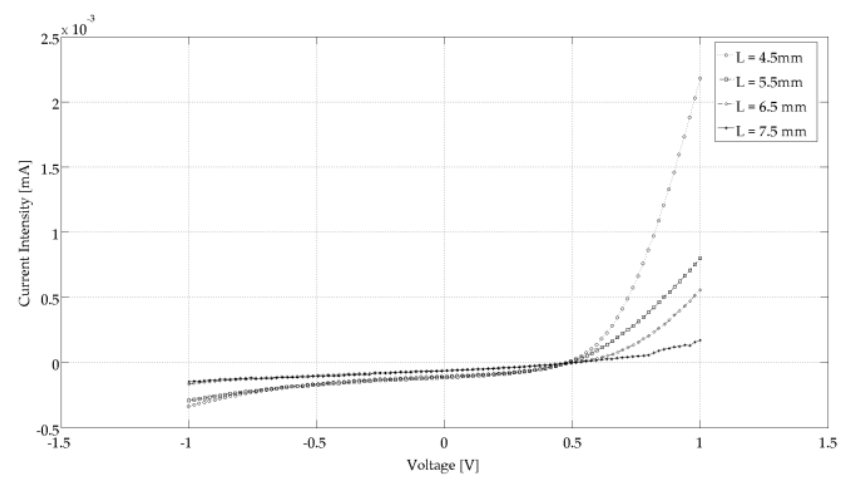

Fig. 3. Current density vs voltage characteristics of organic solar cells with different lengths under 1 sun $\left(100 \mathrm{~mW} / \mathrm{cm}^{2}\right)$ simulated AM1.5 irradiation.

All devices have the same size (width and length of 12 $\mathrm{mm}$ ), while the organic solar cells have a width of $1.5 \mathrm{~mm}$ and a length ranging from 4.50 to $7.50 \mathrm{~mm}$. Fig. 2 shows the realized devices. The I-V measurements are performed under AM 1.5G solar irradiation using a sun light source (K.H. Steuernagel Lichttechnik GmbH solar simulator).

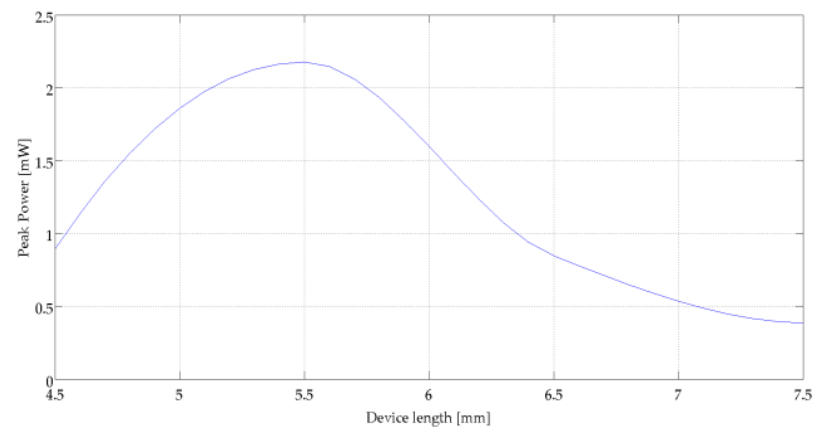

Fig. 4. The relationship between length and maximum power output of the organic solar cells obtained by the selected neural network.

The I-V characteristics were measured with a probe station and parameter analyzer (Keithley Model 2420 SourceMeter instrument). Measurements of emission spectrum were taken using a Stellar Net inc spectrometer. The current density vs voltage characteristics of some organic solar cells with different lengths are shown in Fig. 3. The inputs to the neural network are the different lengths of organic solar cells and the targets are the maximum power outputs associated with each solar cell. The obtained results show that the length of devices has a great influence on their efficiency (see Fig. 4) and that only for a limited subset of organic cell lengths the electric performance of the cells (in this case: in the subset $5.2 \div 5.8 \mathrm{~mm}$ ) is satisfactory. The durability of OPV devices based on fullerene-derived acceptors depends on incident light intensity, temperature of the device during the experiment and the atmosphere (i.e. humidity, oxygen content, glove box, vacuum etc). These phenomena cause macroscopic physical defects on the surface of organic devices such as delamination, formation of particles, bubbles, and cracks, as observed in the Surface Electronic Microscopy (SEM). In this regard, the detection of defects on the surface by modern artificial intelligence and deep learning based on computer vision technology are well suited, during the production cycle, for automating material quality inspection.

The degradation mechanisms of the active inter-layers are fast induced by diffusion of molecular oxygen and water causing chemical reactions in the device, electrode reaction with organic materials, morphological changes due to temperature, and macroscopic changes such as delamination, formation of particles, bubbles, cracks and scratches due to fabrication process at the interfaces of OSCs, as shown in Fig. 5. 

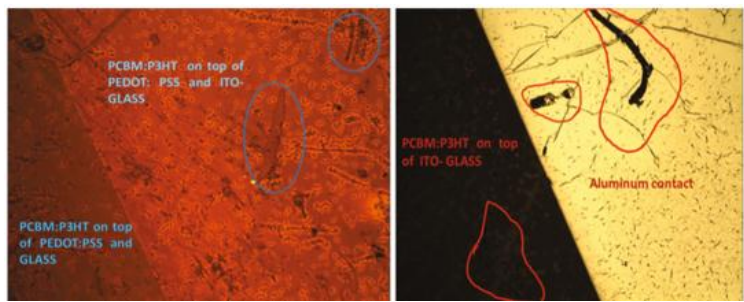

Fig. 5. Defects on interface PCBM:P3HT on top of ITOGlass/PEDOT:PSS and Glass (on left) and on interface PCBM:P3HT on top of ITO-Glass and Aluminum (on right).

The investigation and identification of defects were conducted, such as scratches at different stages of fabrication, or trapped microscopic bubbles during the spin coating stage, on surface morphology of OSCs, and examined by microscopy - Zeiss $4.0 \times$, at $150 \times$ magnification, acquiring 240 images with a resolution of $1280 \times 1024$. The most common type of texture descriptive method is Color Mapping Co-occurrence Matrix (GLCM). GLCM is a square matrix where the number of rows and columns is equal to the number of grey levels in the image, which can reveal certain properties of spatial distribution of grey-levels in the image texture [4]-[5].

The matrix shows how the pixel value $l_{1}$ of a reference pixel occurs in a specific relationship to its neighbouring pixel with a pixel value $1_{2}$. So, each element $\left(l_{1}, l_{2}\right)$ of the matrix represents the number of occurrences of the pair of pixels with pixel values $l_{1}$ and $l_{2}$, which are at a relative distance $\mathrm{d}$ from each other. Mathematically, the elements of the $L \times L$ grey-level co-occurrence matrix with the displacement vector $d(=d i, d j)$, for a given image $I$ of the size $n \times n$ are defined as:

$$
M\left(l_{1}, l_{2}\right)=\sum_{i, j=1}^{n}\left\{\begin{array}{lc}
1 & \text { if } I(i, j)=l_{1} \text { and } I\left(i+d_{i}, j+d_{j}\right)=l_{2} \\
0, & \text { otherwise }
\end{array}\right.
$$

Co-occurrence matrices are calculated for each image. For each channel (Red, Green, Blue) co-occurrence matrices for $\mathrm{d}=1,2$ and in four main directions: $0^{\circ}, 45^{\circ}$, $90^{\circ}$ and $135^{\circ}$ were calculated. In order to describe the texture, a color mapping co-occurrence matrix (GLCM) is usually adopted, leading, however, to dimensionality, and singular value decomposition is used to reduce redundancy arising from texture description by means of GLCM.

For signal and noise separation, SVD and each cooccurrence matrix were introduced taking 12 largest singular values, thus obtaining for each image a feature vector of 216 elements. To recognize defective and nondefective devices, a probabilistic neural network (PNN) was used as shown in Fig. 6, where $\mathrm{X}$ represents the extracted feature vector. The artificial neural network consists of 21 neurons (13 of them represent the defective device and 8 represent the devices with no defects). And once the optimal parameters of the network are obtained, a trained algorithm is applied to classify the data points in the testing dataset into one of the two classes (defective and non-defective devices).

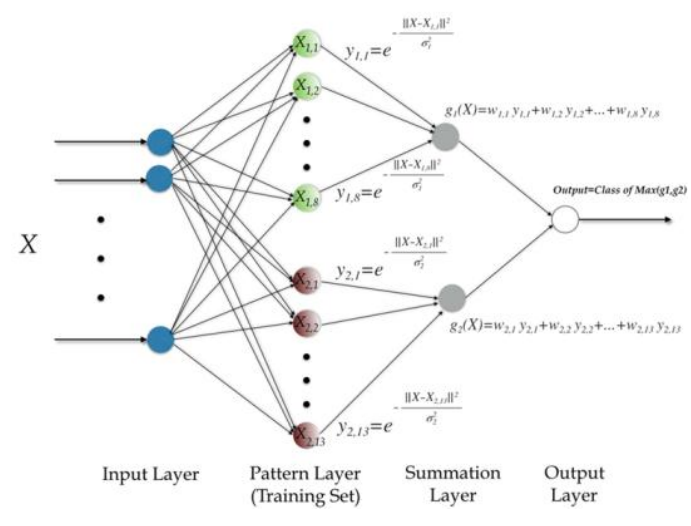

Fig. 6. Adopted Artificial Neural Network

The experimental results show that the algorithm achieves a high recognition accuracy of $96 \%$.

In conclusions, two methodologies based on modern artificial intelligence are described in this paper, useful in optimizing the fabrication process of organic solar cells and making the production workflow more efficient and, therefore, solar cells more competitive and efficient.

The author is grateful for the research and laboratory facilities provided by Optoelectronic Organic Semiconductor Device Laboratory (OOSDL) headed by Prof. Rafi Shikler, Ben-Gurion University of the Negev in Beer Sheva, Israel.

\section{References}

[1] G. Lo Sciuto, G. Capizzi, S. Coco, R. Shikler, Geometric shape optimization of organic solar cells for efficiency enhancement by neural networks, Lecture Notes in Mechanical Engineering, pp. 789796 (2015)

[2] S.N. Barnea, G. Lo Sciuto, N. Hai, R. Shikler, G. Capizzi, M. Wozniak, D. Polap, Photo-electro characterization and modeling of organic light-emitting diodes by using a radial basis neural network, Lecture Notes in Computer Science (including subseries Lecture Notes in Artificial Intelligence and Lecture Notes in Bioinformatics), 10246 LNAI, pp. 378-389 (2017).

[3] L. Ye, H. Hu, M. Ghasemi, T. Wang, B.A. Collins, J.H. Kim, K. Jiang, J.H. Carpenter, H. Li, Z. Li et al., Nat. Mater. 17, 253 (2018).

[4] R.M. Haralick, K. Shanmugam, I. Dinstein, IEEE Trans. Syst. Man. Cybern. SMC-3(6), 610 (1973).

[5] G. Capizzi, G.L. Sciuto, C. Napoli, E. Tramontana, M. Wozniak, Automatic classification of fruit defects based on co-occurrence matrix and neural networks in Federated Conference on Computer Science and Information Systems (FedCSIS), pp. 861-867 (2015). 